SuBMtTted to the Astrophysical Journal.

Preprint typeset using LATEX style emulateapj v. 6/22/04

\title{
BULK COMPTONIZATION OF THE COSMIC MICROWAVE BACKGROUND BY EXTRAGALACTIC JETS AS A PROBE OF THEIR MATTER CONTENT
}

\author{
Markos Georganopoulos $^{1,2}$, Demosthenes Kazanas ${ }^{2}$, Eric Perlman ${ }^{2}$, Floyd W. Stecker ${ }^{2}$ \\ Submitted to the Astrophysical Journal.
}

\begin{abstract}
We propose a method for estimating the composition, i.e. the relative amounts of leptons and protons, of extragalactic jets which exhibit Chandra - detected knots in their kpc scale jets. The method relies on measuring, or setting upper limits on, the component of the Cosmic Microwave Background (CMB) radiation that is bulk-Comptonized by the cold electrons in the relativistically flowing jet. These measurements, along with modeling of the broadband knot emission that constrain the bulk Lorentz factor $\Gamma$ of the jets, can yield estimates of the jet power carried by protons and leptons. We provide an explicit calculation of the spectrum of the bulk-Comptonized (BC) CMB component and apply these results to PKS 0637 - 752 and 3C 273, two superluminal quasars with Chandra detected large scale jets. What makes these sources particularly suited for such a procedure is the absence of significant non-thermal jet emission in the 'bridge', the region between the core and the first bright jet knot, which guarantees that most of the electrons are cold there, leaving the BC scattered $\mathrm{CMB}$ radiation as the only significant source of photons in this region. At $\lambda=3.6-8.0 \mu \mathrm{m}$, the most likely band to observe the BC scattered CMB emission, the Spitzer angular resolution $\left(\sim 1^{\prime \prime}-3^{\prime \prime}\right)$ is considerably smaller than the 'bridges' of these jets $\left(\sim 10^{\prime \prime}\right)$, making it possible to both measure and resolve this emission.
\end{abstract}

Subject headings: galaxies: active - quasars: general - quasars: individual (PKS 0637-752, 3C 273) - radiation mechanisms: nonthermal - X-rays: galaxies

\section{INTRODUCTION}

One of the primary issues that impedes our understanding of extragalactic jets is that of their composition. The power and momentum transferred by the jets from their point of origin, near the central black hole at distance $R_{g} \sim 10^{14} \mathrm{~cm}$, to the radio lobes at Mpc scales is transported by an electrically neutral, yet unspecified combination of leptons (both electrons and positrons or $e^{ \pm}$), protons, and Poynting flux carried by the magnetic field of the flow. For a given radiative output, the jet power depends on the composition of the outflowing matter, with leptonic $\left(e^{ \pm}\right)$jets demanding less overall power and mass and being easier to accelerate to relativistic bulk flow velocities than hadronic $(e-p)$ ones. Uncertainty in the matter content results in uncertainty in the jet power, which bears on our understanding of the jet dynamics and the radio lobe energetics, their interaction and influence on the host galaxy and possibly the embedding cluster core (e.g. Omma \& Binney 2004). The composition is also related to the fundamental problem of jet formation: the plasma in jets powered by an accretion disk (Blandford \& Payne 1982; Königl 1989) would be expected to be baryon loaded, while jets powered by the rotational energy of a black hole are more likely to result in $e^{ \pm}$jets (Blandford \& Znajek 1977).

A number of attempts have been made over the years toward measuring, or at the least constraining, the matter content of jets, in particular the fraction of kinetic energy stored in protons and low energy or cold leptons, whose low radiative efficiencies fail to provide direct ev-

${ }^{1}$ Department of Physics, Joint Center for Astrophysics, University of Maryland-Baltimore County, 1000 Hilltop Circle, Baltimore, MD 21250, USA

2 Laboratory for High Energy Astrophysics, NASA Goddard Space Flight Center, Code 661, Greenbelt, MD 20771, USA. idence of their presence. To this end, a variety of argutments have been presented, based on the synchrotron self Compton (SSC) formalism (e.g. Celotti \& Fabian 1993; Reynolds et al. 1996; Hirotani 2004), on the circular polarization of the radio emission measured in 3C 279 and other sources (e.g. Wardle et al. 1998), and on the pressure balance of the radio lobes and the X-ray emitting confining plasma (e.g., Gizani \& Leahy 2004). These largely indirect arguments have produced rather contradictory results, with different works supporting leptonic and hadronic contributions of varying proportions to the jet content.

A direct estimate of the cold lepton content of blazar jets was proposed by Begelman \& Sikora (1987), Sikora \& Madejski (2000), and Modersksi et al. (2004). The gist of their argument is the following: The observed nonthermal blazar emission is thought to be produced at distances $\sim 10^{17}-10^{18} \mathrm{~cm}$ from the central engine (e.g. Sikora 1997); the jet leptons providing the blazar emission at these distances need to be transported practically cold by a relativistic flow of bulk Lorentz factor $\Gamma \sim 10$ from the black hole vicinity to the blazar emission site; as these cold jet leptons propagate through the quasar broad line region (BLR) they would Compton - scatter the BLR optical-UV photons (of energy $E_{\text {O-UV }} \sim 10$ $\mathrm{eV})$ to energies $E_{\mathrm{BC}} \simeq \Gamma^{2} E_{\mathrm{O}-\mathrm{UV}} \simeq 1 \mathrm{keV}$, to produce a black-body type hump in their X-ray spectra. The fact that such a feature has not been observed in the inverseCompton dominated X-ray spectrum of blazars was used by the above authors to conclude that the power in these jets is carried mainly by protons, although cold leptons dominate the number of particles in the jet. While this idea is well founded and appealing, concrete answers are hindered by unknowns such as the distance at which the jet is formed, its sub-pc scale opening angle and the ac- 
tual photon energy density of the BLR, as well as by the presence of a strong X-ray non-thermal continuum that apparently could "hide" the proposed bulk-Comptonized component.

Arguments based on the bulk Compton (BC) emission used by the above authors can be applied to any astrophysical site involving relativistic flows. One can then obtain more concrete conclusions provided that the flow geometry and the target photon density are better determined. Such a site is presented by the large scale (100's of $\mathrm{kpc}$ ) jets of superluminal quasars such as PKS 0637752 (Schwartz et al. 2000; Chartas et al. 2001) and 3C 273 (Marshall et al. 2001; Sambruna et al. 2001; Jester et al. 2002) detected by the Chandra X-ray Observatory. Such large scale jets are tightly collimated and propagate through the very well understood CMB. The effect of the CMB scattering by the jet cold electrons is akin to the well known SunyaevZeldovich (SZ) effect associated with clusters of galaxies, with the difference being that in this case the CMB photons are scattered by electrons moving with a bulk relativistic velocity (see below) rather than by thermal electrons.

These objects and their jets have morphologies which are conducive to applying the arguments referred to above. They exhibit radio, optical, and X-ray emission from the quasar core and then from well separated knots along the jet at angular distances $\sim 8^{\prime \prime}$ for PKS 0637752 and $\sim 13^{\prime \prime}$ for $3 \mathrm{C} 273$. The region between the core and the first knot, which we will refer to as the 'bridge' in the rest of this work, is very weakly radiating in radio, optical, and X-ray energies. This is very important because: ( $i$ ) It shows that most of the leptons propagating through the 'bridge' are 'cold', since there is only weak synchrotron and inverse Compton emission from this section of the jet. (ii) It provides a region free from unwanted contamination by unrelated broad band nonthermal radiation. As in the $\mathrm{SZ}$ effect, where the $\mathrm{X}$-ray cluster properties provide values of the parameters involved in the scattering of the CMB photons, the jet properties (power and kinematics) are provided by the properties (spectrum and luminosity) of the Chandra - detected knots of these jets.

Schwartz et al. (2000) noted that the X-ray emission from the jet knots detected by Chandra at a projected distance of $\sim 70-100 \mathrm{kpc}$ from the nucleus of PKS 0637 752 is part of a spectral component separate from the knot radio-optical synchrotron emission and that it is too bright to be explained through SSC emission from electrons in energy equipartition with the jet magnetic field. Observational evidence now indicates that this property is common to the jets of many other quasars (Sambruna et al. 2002, 2004; Siemiginowska et al. 2002, 2003; Marshall et al. 2004; Jorstad \& Marscher 2004; Yuan et al. 2003; Cheung 2004). Tavecchio et al. (2000) and Celotti et al. (2001) proposed that the X-ray emission is due to external Compton (EC) scattering of $\mathrm{CMB}$ photons off relativistic electrons in the jet, provided that the jet flow is sufficiently relativistic $(\Gamma \sim 10)$ to boost the $\mathrm{CMB}$ energy density in the flow frame (by $\Gamma^{2}$ ) to the level needed to reproduce the observed X-ray flux. This was the first suggestion, based on extended X-ray emission, that powerful jets retain significantly relativistic veloci- ties at large distances from the core, a very important feature because it boosts the level of the anticipated BC emission by $\sim \mathrm{I}^{2}$.

Here we argue that our recently gained understanding that the Chandra-detected quasar jets remain relativistic on scales of hundreds of kpc, together with the IR capabilities of Spitzer, can be used to measure or substantially constrain the matter content of these jets. In $\S 2$ we calculate the $\mathrm{BC}$ spectrum of the $\mathrm{CMB}$ as a function of the cold lepton kinetic power and Lorentz factor $\Gamma$, as well as its polarization. In $\S 3$ we present the mechanisms that have been proposed to explain the X-ray knot emission, and we argue that EC off the CMB is the most probable mechanism. In $\S 4$ we use the simple analytical arguments of Dermer \& Atoyan (2004a; hereafter DA04) to derive the flow velocity and estimate the number of electrons carried by the jet. We then apply our calculations to the quasars PKS 0637-752 and $3 \mathrm{C} 273$ in $\S 5$. Finally, in $\S 6$ we discuss our findings along with some caveats and present our conclusions.

\section{BULK COMPTON SPECTRUM AND POLARIZATION}

The expected level of $\mathrm{BC}$ emission depends on the jet power in cold leptons, its length, its Lorentz factor $\Gamma$ and its angle to the observer's line of sight. We now proceed to calculate the spectrum and luminosity of the radiation produced by the propagation of a collimated cold electron beam (mean electron Lorentz factor $\langle\gamma\rangle \approx 1$ ) of Lorentz factor $\Gamma$ and power $L_{e}$ through an isotropic photon field. We start with a simple estimate of the peak energy and peak luminosity as a function of observing angle assuming a monoenergetic photon field with dimensionless energy $\epsilon_{0}$ (in units of $m_{e} c^{2}$ ) and energy density $U$. Assuming that the jet has a length $l$, the fractional energy loss of a single electron after propagating this distance $l$ is

$$
\frac{\Delta \Gamma}{\Gamma}=\frac{4}{3} \frac{\sigma_{\mathrm{T}}}{m_{e} c^{2}} \beta \Gamma U l,
$$

where $\beta$ is the beam velocity in units of $c$ and $\sigma_{\mathrm{T}}$ is the Thomson cross section. Given that the CMB photon energy density $U$ scales with redshift $z$ as $U=U_{0}(1+z)^{4}$, where $U_{0}=4.1810^{-13} \mathrm{erg} \mathrm{cm}^{-3}$, for a large scale quasar jet propagating through the $\mathrm{CMB}$ the fractional energy losses are

$$
\frac{\Delta \Gamma}{\Gamma} \approx 1.410^{-6} \Gamma_{10} l_{100 K p c}(1+z)^{4} .
$$

Therefore, the bulk deceleration due to Compton drag off the CMB can be safely ignored for $z \lesssim 10$, even for the extreme case of a purely leptonic large scale jet.

The inferred isotropic BC luminosity $L_{B C}$ at a given observing angle $\theta$ is given by the fractional electron losses multiplied by $L_{e}$ and by the beaming pattern $\delta^{3} / \Gamma$ of a continuous flow (e.g. Sikora et al. 1997),

$$
L_{B C}=L_{e} \frac{\Delta \Gamma}{\Gamma} \frac{\delta^{3}}{\Gamma}=\frac{4}{3} \frac{\sigma_{T}}{m_{e} c^{2}} L_{e} \beta U l \delta^{3},
$$

where $\delta=1 /[\Gamma(1-\beta \cos \theta)]$ is the well known Doppler factor. The observed luminosity peaks at

$$
\epsilon_{B C} \approx 2 \epsilon_{0} \Gamma \delta \text {. }
$$

If we assume $\theta=1 / \Gamma$, which corresponds to $\delta=\Gamma$, the $\mathrm{BC}$ emission has a power

$$
L_{B C} \approx 1.410^{-4} l_{100 K p c} \Gamma_{10}^{3}(1+z)^{4} L_{e}
$$


and peaks in the IR regime, $\nu_{B C} \approx 410^{13} \Gamma_{10}^{2} \mathrm{~Hz}$. For constant Lorentz factor jets, this emission due to the $\mathrm{BC}$ scattering of the $\mathrm{CMB}$ will be evenly distributed between the core and the first knot, which for $\theta=1 / \Gamma$ will have a projected length $l / \Gamma$, and a luminosity per unit projected length scaling as $\Gamma^{4}$. Note that: (i) the BC surface brightness is independent of $z$, because its cosmological decrease by $(1+z)^{-4}$, is exactly compensated by the increase of the CMB energy density by $(1+z)^{4}$ (Schwartz 2002). (ii) The observed peak frequency is also independent of $z$ because while the CMB photon energy and therefore the peak emission energy scales as $(1+z)$, the received photon energy scales as $1 /(1+z)$. (iii) The independence of the surface brightness of the BC component on the redshift $z$ and the fact that the angular size of an object is roughly constant for $z \gtrsim 1$, imply that the flux of this feature will also remain constant independent of the source redshift (Schwartz 2002), in distinction to the core and remaining synchrotron jet emission that decrease significantly with increasing $z$.

The Spectrum. To derive the spectrum of the $\mathrm{BC}$ emission off the CMB, we extend the calculation of section $\$ 7.3$ of Rybicki \& Lightman (1979) from a monoenergetic to a blackbody photon field. The intensity of the blackbody photon field in terms of number of photons is:

$$
I(\epsilon)=F_{0} \frac{\epsilon^{2}}{\exp \left(\epsilon / \epsilon_{0}\right)-1}, F_{0} \equiv \frac{2 m_{e}^{3} c^{4}}{h^{3}}, \epsilon_{0} \equiv k T / m_{e} c^{2}
$$

where $T$ is the blackbody temperature. Using the invariance of $I / \epsilon^{2}$, we obtain the incident intensity field in the (primed) frame of the electron beam:

$$
I^{\prime}\left(\epsilon^{\prime}, \mu_{0}^{\prime}\right)=F_{0} \frac{\epsilon^{\prime 2}}{\exp \left[\epsilon^{\prime} \Gamma\left(1+\beta \mu_{0}^{\prime}\right) / \epsilon_{0}\right]-1},
$$

where $\mu_{0}^{\prime}=\cos \theta_{0}^{\prime}$ and $\theta_{0}$ is the angle the incoming photons form with the direction of the electron motion. The emission function in the beam frame, assuming isotropic Thomson scattering with $d \sigma^{\prime} / d \Omega_{0}^{\prime}=\sigma_{\mathrm{T}} / 4 \pi$, is

$$
j^{\prime}\left(\epsilon_{1}^{\prime}\right)=\frac{1}{2} n^{\prime} \sigma_{\mathrm{T}} \int_{-1}^{+1} I^{\prime}\left(\epsilon^{\prime}, \mu_{0}^{\prime}\right) d \mu_{0}^{\prime},
$$

where $n^{\prime}$ is the electron number density in the beam frame. Performing the integration, we obtain:

$$
j^{\prime}\left(\epsilon_{1}^{\prime}\right)=\frac{n^{\prime} \sigma_{\mathrm{T}} F_{0} \epsilon_{0}}{2 \beta \Gamma} \epsilon_{1}^{\prime} \ln \frac{1-\exp \left[-\epsilon_{1}^{\prime} \Gamma(1+\beta) / \epsilon_{0}\right]}{1-\exp \left[-\epsilon_{1}^{\prime} /\left(\epsilon_{0} \Gamma(1+\beta)\right)\right]} .
$$

Using the invariance of $j / \epsilon$ we obtain:

$j\left(\epsilon_{1}, \mu\right)=\frac{\epsilon_{1}}{\epsilon_{1}^{\prime}} j^{\prime}\left(\epsilon_{1}^{\prime}\right)=\frac{n^{\prime} \sigma_{\mathrm{T}} F_{0} \epsilon_{0}}{2 \beta \Gamma} \epsilon_{1} \ln \frac{1-\exp \left[-\epsilon_{1} \Gamma^{2}(1+\beta)(1\right.}{1-\exp \left[-\epsilon_{1}(1-\beta \mu) /\left(\epsilon_{0}\right.\right.}$

The power of the cold electron beam is $L_{e} \approx S \beta c \Gamma^{2} n^{\prime}$, where $S$ is the jet cross section. Using this in the above equation and multiplying by the beam volume $V=S l$, by the final photon energy $\nu=m_{e} c^{2} \epsilon_{1} / h$, and by $4 \pi$, we obtain the $\mathrm{BC}$ specific luminosity of the source

$$
L_{\nu}=\frac{L_{e} \sigma_{\mathrm{T}} l k T}{m_{e} c^{5} \beta^{2} \Gamma^{3}} \nu^{2} \ln \frac{1-\exp [-h \nu \Gamma(1+\beta) /(\delta k T)]}{1-\exp [-h \nu /(\Gamma \delta k T(1+\beta))]},
$$

where we have also used the definition of $\delta$ and the relation $\epsilon_{1} / \epsilon_{0}=h \nu / k T$. As can be seen in Figure 1 the simple estimates of the luminosity and peak frequency agree relatively well with the results of the spectral calculation, and can be safely used for simple estimates.

The Polarization. The emission we consider, being the result of Compton scattering, is expected to be polarized, perhaps very highly so, since in the jet flow rest frame the seed photons are essentially unidirectional. The polarization can be easily calculated in the approximation of a perfectly collimated cold electron beam with $\Gamma \gg 1$ (for a general treatment of BC emission polarization see Begelman \& Sikora 1987). The degree of polarization $\Pi$ is a Lorentz invariant and at the beam frame can be written as $\Pi=\left(1-\mu^{2}\right) /\left(1+\mu^{\prime 2}\right)$. Using the light aberration relation $\mu^{\prime}=(\mu-\beta) \Gamma \delta$, one can obtain immediately the degree of polarization $\Pi$ in the observer's frame:

$$
\Pi=\frac{1-(\mu-\beta)^{2} \Gamma^{2} \delta^{2}}{1+(\mu-\beta)^{2} \Gamma^{2} \delta^{2}}
$$

As can be seen in Figure 2 the degree of polarization is a very sensitive function of orientation, reaching $100 \%$ at $\theta=1 / \Gamma$ and dropping rapidly to $0 \%$ at $\theta=0$. Using the above expression along with the relation $\epsilon_{B C}=2 \Gamma \delta \epsilon_{0}$ between the initial $\epsilon_{0}$ and final $\epsilon_{B C}$ photon energies, one can obtain the values of both $\beta$ (or equivalently $\Gamma$ ) and $\theta$ in terms of the observables $\Pi$ and $\epsilon_{B C}$. Setting $A^{2}=$ $(1-\Pi) /(1+\Pi)$ and $B=2 \epsilon_{0} / \epsilon_{B C}$, we obtain

$$
\beta=\frac{-A B \pm \sqrt{A^{2} B^{2}+4(1-B)}}{2}, \mu=\cos \theta=A B+\beta,
$$

where the positive sign corresponds to an approaching beam. Such polarization measurements of the BC emission can in principle be performed in the near IR to provide an independent measurement of both the Lorentz factor $\Gamma$ and the angle $\theta$ of the jet to the observer's line of sight that can break the degeneracy normally present in, e.g., VLBI measurements.

\section{THE X-RAY EMISSION MECHANISM}

The $\mathrm{BC}$ emission of the $\mathrm{CMB}$ in the 'bridge' region of a jet flow depends on the power carried by cold leptons, the flow Lorentz factor $\Gamma$ and the angle of the jet to the observer's line of sight $\theta$, as shown by Equation (3). Estimates or constraints on these quantities are provided by the non-thermal emission at the knot marking the end of the 'bridge'. The Chandra detections are critical in providing such estimates. This brings on the issue of the X-ray emission process in the knots of the Chandradetected quasar jets, as different mechanisms produce different constraints for the quantities on which the level of $\mathrm{BC}$ emission depends. While EC of the CMB (Tavec-

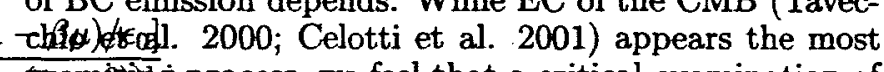
$\epsilon_{0}$ promising process, we feel that a critical examination of the alternatives is at this point necessary.

Dermer \& Atoyan (2002) suggested that the observed $\mathrm{X}$-rays are due to synchrotron emission from electrons cooling by EC off the CMB in the Klein-Nishina (KN) regime. Due to the reduced KN-losses of the highest energy electrons on the thermal CMB spectrum, their distribution function develops a "hump" at these energies, which manifests itself in the synchrotron emission of these electrons as an increase in the spectral luminosity of this component between UV and X-ray energies. The continuity of the electron distribution function, then, implies also the continuity of the spectrum between optical, 
UV, and X-rays. As a result, the extrapolation of the observed X-ray spectrum to lower frequencies, namely UV and optical, must always lie below the observed UV and optical fluxes. This is actually contrary to observation, as indicated by the optical detections or upper limits at several such knots (e.g. PKS 0637-752, Chartas et al. 2000; Knot A of $1354+195$, Sambruna et al. 2004; Knot B of 1150-089, Sambruna et al. 2002; Knot C4 of $0827+243$, Jorstad \& Marscher 2004). This interpretation could be valid, however, for the X-ray emission of knot $\mathrm{A}$ of $3 \mathrm{C}$ 273 , as the near-IR - optical -UV spectrum indeed turns upward, pointing toward the X-ray point (Jester et al. 2002).

Alternatively, it has been suggested (e.g. Schwartz 2000) that the $X$-rays are due to synchrotron radiation by a second, very energetic electron population with a low energy cut-off at sufficiently high energy that its synchrotron emission results in a low energy cut-off at UV energies to comply with observation. However, even if an unknown mechanism can produce the injection of an election population with the above properties, they would cool in less than a knot-crossing time to energies below those corresponding to optical synchrotron emission. This fact has two unfavorable implications: (i) Given the observed X-ray spectral indices $\left(\alpha_{x}=(\gamma-1) / 2 \sim\right.$ $0.5-0.8$; e.g. Sambruna et al. 2004), the injected electron distribution must have an index $p$ flatter by one unit than that observed, i.e., $p=\gamma+1=2 \alpha_{x} \sim 1-1.6$ (see also Aharonian 2002), significantly flatter than the asymptotic values predicted by particle acceleration theories ( $p \simeq 2-2.3$, e.g. Kirk et al. 2000). (ii) These high energy electrons will cool below their low energy cut-off to produce, in this energy range, an electron distribution $N_{e}(\gamma) \propto \gamma^{-2}$; the synchrotron emission of these electrons would then lead to a $\nu^{-1 / 2}$ spectrum that in many cases, such as PKS 0637-752, overproduces the observed optical fluxes. These problems can be overcome if instead of a simple injection one invokes the continuous acceleration of the radiating electrons in multiple shocks or spatially distributed stochastic acceleration (Stawarz et al 2004). In both cases such models can produce a pile-up of high energy electrons at the upper end of the electron distribution which could lead to X-ray synchrotron consistent with observations. These models, like that of Dermer \& Atoyan (2002), can only model successfully emission by sources in which the extrapolation of the X-ray spectrum to optical frequencies lies below the observed optical flux, such as the knot $A$ of $3 C 273$.

The EC interpretation of the knot X-ray emission is not entirely without problems either. Multiwavelength observations have shown that in many cases the emission profiles at the knot regions are largely achromatic. This behavior is unexpected (Tavecchio, Ghisellini, \& Celotti 2003; Stawarz et al. 2004), because the cooling length of the EC X-ray emitting electrons ( $\gamma \sim$ few hundreds) is longer than that of the radio emitting ones $(\gamma \sim$ few thousands) and comparable to or longer than the size of the entire jet; this would lead one to expect longer jet emission in X-rays than that in the radio.
However, in most Chandra-detected quasar jets, the radio-to-X-ray logarithmic slope $\alpha_{r x}$ increases downstream along the jet. Indeed, some (e.g. 3C 273 in Sambruna et al. 2001 and Marshall et al. 2001; PKS 1136135 and $1354+195$ in Sambruna et al. 2002; PKS 1127145 in Siemiginowska et al. 2002;0827+243 in Jorstad \& Marscher 2004) show anti-correlated X-ray and radio maps, with the $\mathrm{X}$-ray emission peaking closer to the core, gradually decreasing outward, while the radio emission increases outward to peak practically at the jet terminus. This problem is alleviated, however, if the large-scale jet gradually decelerates (Georga-nopoulos \& Kazanas 2004) downstream from the first knot. Then, the X-ray brightness decreases along the jet because the CMB photon energy density in the flow frame decreases. At the same time, the deceleration leads to an increase of the magnetic field in the flow frame, which enhances the radio emission with distance. As a result the radio emission is shifted downstream of the $\mathrm{X}$-rays and the radio to $\mathrm{X}$-ray spectral logarithmic slope $\alpha_{r x}$ increases along the jet, in agreement with observations. The notion of relativistic and decelerating flows in the large scale quasar jets is in agreement with the recent suggestion (Georganopoulos \& Kazanas 2003) that the flow in the terminal hot spots of powerful jets must also be mildly relativistic $(\Gamma \sim 2-3)$ and decelerating to sub-relativistic velocities.

In conclusion, we consider the EC model (Tavecchio et al. 2000; Celotti et al. 2001), with the modification of the bulk flow deceleration proposed by Georganopoulos \& Kazanas (2004) to be the most favorable process accounting for the observed knot emission in this class of sources. However, the synchrotron interpretation for the $\mathrm{X}$-ray emission is still viable, at least for the sources consistent with a continuous underlying electron distribution, manifest by the conituity between the optical to X-ray spectra, as discussed above. This possibility will be considered in the determination of the jet properties of $3 \mathrm{C}$ 273.

\section{THE JET POWER}

Having discussed the mechanism responsible for the $\mathrm{X}$-ray emission of the jet knots, we now turn to the determination of their dynamical parameters subject to the constraint that they account for the observed X-ray emission of the knots. These estimates are based principally on the energetics of the emission rather than the details of the spectra, as the latter can be reproduced by appropriate choice of additional parameters pertaining to the particle distributions. However, even at this level, the number of parameters exceeds that of the observables. One therefore resorts to minimum energy arguments in order to further constrain the available values of the magnetic field $B$, the Doppler factor $\delta$ and the energy flux in or the power of the jet flows.

A comprehensive set of constraints for the jet power and beaming based on multiwavelength observations of knots in the extended jet has been recently presented by DA04 (see also Ghisellini \& Celotti 2001). These authors produced analytic relations for the jet Doppler factor $\delta_{\min }$ that minimizes the jet power and are consistent with all constraints imposed by the knot multiwavelength emission. In this respect one should note that, because the quantity that is minimized is the total knot power 
and not the power in relativistic electrons and magnetic field only, the value of $\delta_{\min }$ depends on the matter content of the jet.

\subsection{Minimum Jet Power for Knot X-ray Emission Due to $E C$}

The work of DA04 models the knots as homogeneous sources moving with a Lorentz factor $\Gamma$ at an angle $\theta$ to the line of sight. The knot matter content is described through the ratio $k_{p e}$ of the power carried by protons to the power carried by leptons in the knot. For a pure $e^{ \pm}$ composition, $k_{p e}=0$, while for an $e-p$ jet this parameter reaches its maximum value of

$$
k_{p e}=\frac{m_{p}(p-2)}{m_{e} \gamma_{\min }(p-1)},
$$

where $\gamma_{\min }$ and $p(>2)$ are the minimum Lorentz factor and energy index of the power law electron energy dis tribution (EED), and the protons are considered to be cold in the knot comoving frame (note that DA04 use $k_{p e}=m_{p} /\left(m_{e} \gamma_{\min }\right)$, valid only for $\left.p \gg 1\right)$. Assuming that the $\mathrm{X}$-rays are due to EC scattering off the CMB and that $\delta=\Gamma$, DA04 calculate (their Eq. (12)) the Doppler factor $\delta_{\min }$ that minimizes the power that has to be supplied to the knot by the jet. Their expression can be written as

$$
\delta_{\min }=f_{1}\left(\frac{1+k_{p e}}{\gamma_{\min }^{p-2}}\right)^{1 /(5+p)},
$$

where $f_{1}$ depends on the source redshift, the radio and $\mathrm{X}$-ray fluxes and spectral index (the radio and X-ray index are assumed to be the same, as would be the case if they are due to a single power-law electron distribution) and the linear size of the knot $\left(f_{1}\right.$, along with the subsequently used $f_{2}, f_{3}, f_{4}$ are reproduced in Appendix A). In particular, as can be seen from Eq. (14) and (15), the ratio of hadronic to leptonic Doppler factors depends only on $\gamma_{\min }$ and $p$ :

$\frac{\delta_{\min , e-p}}{\delta_{\min , e^{ \pm}}}=\left(1+k_{p e}\right)^{1 /(5+p)} \approx\left[\frac{m_{p}(p-2)}{m_{e} \gamma_{\min }(p-1)}\right]^{1 /(5+p)}$, , for

The lowest possible value for $\gamma_{\min }$ is constrained by the requirement that the EC emission does not extend to frequencies as low as optical. Similarly the maximum possible value for $\gamma_{\min }$ is constrained by the requirement to be sufficiently small that its Comptonization of the CMB leads to the observed X-ray emission. In Figure 3 we plot $\delta_{\min }$ as a function of $\gamma_{\min }$ for the extreme cases of an $e^{ \pm}$(solid curve) and an $e-p$ composition (dashed curve) for the knot WK7.8 of PKS 0637-752. The observational parameters needed for the calculation are taken by Chartas et al. (2000) and Schwartz et al. (2000) and can be found in Appendix A. For those values of $\gamma_{\min }$ for which both solutions are permitted, the hadronic knot is characterized by substantially higher $\delta_{\text {min }}$.

We now turn to the minimum knot power $L_{\min }$ that corresponds to $\delta_{\min }$, which, following DA04, is written as:

$$
\begin{gathered}
L_{\min }=L_{p a r t}+L_{B} \\
L_{p a r t}=f_{2}\left(1+k_{p e}\right) \gamma_{\min }^{2-p} \delta_{\min }^{-(1+p)} \\
L_{B}=f_{3} \delta_{\min }^{4}
\end{gathered}
$$

where $L_{\text {part }}$ is the power in particles and $L_{B}$ is magnetic field power, and $f_{2}, f_{3}$ are functions of observables used in DA04 (see Appendix A). In Figure 4, we plot the total minimum knot power as a function of $\gamma_{\min }$ for an $e^{ \pm}$(thin solid curve) and an $e-p$ (thick solid curve), for the case of knot WK7.8 of PKS 0637-752. As can be seen, the minimum power for a hadronic knot flow (equal numbers of $p$ 's and $e$ 's) is always larger than that for a leptonic one. Interestingly, the leptonic power (dashed line) needed to produce the observed emission in minimum total power conditions is larger for a leptonic jet. This is mostly due to the lower Doppler factor $\delta$ of leptonic relative to hadronic knot flows and the strong dependence of the knot emission on $\delta$. In conclusion, for a given choice of $\gamma_{\min }$, the Doppler factor and the jet power are only a function of the matter content, and can be used to calculate the $\mathrm{BC}$ emission using the formalism of $\S 2$.

\subsection{Minimum Jet Power for Knot X-ray Emission Due to Synchrotron}

If we assume a synchrotron interpretation for the broadband knot spectrum, then, as DA04 point out, the power needed to produce the second high energy component is only a small fraction of the power needed to produce the low energy synchrotron emission. This is mostly because the electron radiative efficiency is much smaller at radio energies. Therefore, we focus on the power needed to produce the radio emission. Here, for a given observed synchrotron radio spectrum, the Doppler factor at minimum power conditions cannot be uniquely defined. Instead, it is the product $B \delta$ that can be derived. Following DA04, their Eq. (8) can be written as

$$
\delta \epsilon_{B}=f_{4}\left(\frac{\gamma_{\min }^{2-p}}{\tilde{k}_{e q}}\right)^{2 /(5+p)}
$$

where $\epsilon_{B}$ is the magnetic field in units of the critical magnetic field $B_{c r}=m_{e}^{2} c^{3} / e \hbar, f_{4}$ is a function of ob-

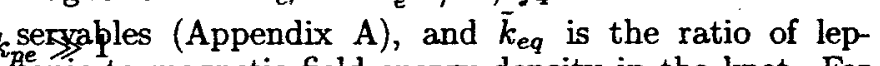
tonic to magnetic field energy density in the knot. For $\bar{k}_{e q}=1, y_{e q}=\left(\delta \epsilon_{B}\right)_{e q}=f_{4} \gamma_{\min }^{2(2-p) /(5+p)}$ and $\delta \epsilon_{B}=$ $y_{e q} / \bar{k}_{e q}^{2 /(5+p)}$. The jet power flowing through the knot is $L=\pi r_{b}^{2} c \delta^{2}\left(u_{\text {part }}+u_{B}\right)$, where $u_{B}$ is the magnetic field energy density, $u_{\text {part }}=\left(1+k_{p e}\right) \bar{k}_{e q} u_{B}$ is the total particle energy density, and we have made the usual assumption $\delta=\Gamma$. The jet power can then be written as

$$
L=\pi r_{b}^{2} c u_{B_{c r}}\left[\left(1+k_{p e}\right) y_{e q}^{(5+p) / 2} y^{-(1+p) / 2}+y^{2}\right]
$$

where $y=\delta \epsilon_{B}$ and it is minimized for

$y_{\min }=\left(\delta \epsilon_{B}\right)_{\min }=f_{4} \gamma_{\min }^{-2(p-2) /(5+p)}\left[\frac{\left(1+k_{p e}\right)(p+1)}{4}\right]^{2 /(5+p)}$.

Setting $y=y_{\min }$ in Equation (19) we can calculate the jet power as a function of $\gamma_{\min }$ and the jet matter content expressed through $k_{p e}$.

\section{APPLICATIONS}

We focus our attention on the Chandra-detected superluminal quasars PKS 0637-752 and 3C 273, whose superluminal nature guarantees that their jets must be at 
relatively small viewing angles $\theta \sim 1 / \Gamma$, where $\Gamma \approx \beta_{a p p}$ is the minimum bulk Lorentz factor that corresponds to the detected superluminal velocity $\beta_{a p p}$ (see e.g. Urry \& Padovani 1995). This suggests actual 'bridge' lengths $\sim \Gamma$ times longer than their projections on the plane of the sky, resulting in separations of hundreds of $\mathrm{kpc}$ between the core and the first knot.

Using the analytic expressions of $\$ 4$ we first estimate the power $L_{\text {lept }}$ in leptons and Doppler factor $\delta$ required to reproduce the emission from the knot at which the radiatively inefficient 'bridge' terminates, under minimum power conditions. We then proceed to calculate the $\mathrm{BC}$ flux using the formalism of $\S 2$ under two different, general assumptions.

Case A: The lepton power $L_{\text {lept }}$ required in the knot is provided by the cold leptons in the flow $\left(L_{e}=L_{\text {lept }}\right)$. This then requires that only a minority of these leptons get accelerated at the knot to create the X-ray producing population by tapping a small fraction of the kinetic energy of the remaining 'cold' leptons. This represents one of the most optimistic cases for detecting the BC CMB emission in the 'bridge' region as it requires a large number of cold leptons in the jet.

Case B: The most conservative case for detecting the anticipated BC emission is that in which the jet provides only the number of leptons needed to produce the observed X-ray emission at knot; the leptons are accelerated there using exclusively the energy of other agents such as the magnetic field and/or the jet hadrons. In this case $L_{e}=L_{\text {lept }}(p-2) /(p-1) \gamma_{m i n}$, and the cold lepton luminosity, and subsequently the $\mathrm{BC}$ emission is lower by a factor of $\gamma_{\min }(p-1) /(p-2)$ compared to case $\mathrm{A}$.

In both cases the jet composition can range from purely leptonic to equal number of electrons and protons. In the following we discuss only these two extreme cases as they bracket all other combinations of protons and leptons. In both cases we assume that the Doppler factor of the flow in the 'bridge' is not significantly different from that derived for the knot. We defer a discussion of this assumption for $\$ 6$.

\subsection{PKS 0637-752}

In the case of PKS 0637-752, the optical flux from knot WK7.8, the knot at which the radiatively inefficient 'bridge' terminates, is clearly below the extrapolation of the X-ray spectrum at lower frequencies, and, as we argued in $\S 3$, this favors the EC interpretation for the $\mathrm{X}$ rays. For knot WK7.8 we adopt $\gamma_{\min }=20$, which, using Equation (15) and the observational data in Appendix A, corresponds to minimum power Doppler factors $\delta_{\min }=$ 17.4 for a leptonic composition and $\delta_{\min }=27.8$ for a hadronic composition, as can be seen in Figure 3. It also corresponds to a jet minimum power $L_{\min }=9.7 \times 10^{45}$ erg $\mathrm{s}^{-1}$ for the leptonic jet and $L_{\min }=6.3 \times 10^{46} \mathrm{erg}$ $\mathrm{s}^{-1}$ for the hadronic jet. The corresponding lepton power is $L_{\text {lept }}=3.7 \times 10^{45} \mathrm{erg} \mathrm{s}^{-1}$ for the leptonic jet and $L_{\text {lept }}=6.8 \times 10^{44} \mathrm{erg} \mathrm{s}^{-1}$ for the hadronic one. As can be seen in Figures 3 and 4, these numbers are only weakly affected by our choice of $\gamma_{\min }$, as long as $\gamma_{\min } \gtrsim 10$. To estimate the actual length of the 'bridge', we make the usual for superluminal sources assumption that the source is observed at an angle $\theta=1 / \Gamma$. At the redshift of the source $(z=0.651), 1^{\prime \prime}$ corresponds to $6.9 \mathrm{Kpc}$ assuming standard cosmology (Spergel et al. 2003) and the deprojected 'bridge' length is $l \approx 930 \mathrm{Kpc}$ for a leptonic jet and $l \approx 1.5 \mathrm{Mpc}$ for a hadronic jet. We are discussing the implications of these length estimates in $\S 6$.

To calculate the BC flux we proceed using the formalism of $\S 2$ for an $e-p$ and an $e^{ \pm}$composition for both cases $A$ and $B$ for the cold lepton power described above. As can be seen in Figure 5, in case $A$ the emission for a leptonic jet peaks at mid IR energies, while that for a hadronic jet peaks at near IR - optical energies. For both compositions the anticipated mid IR flux is above the Spitzer sensitivity limits; the hadronic case however violates the HST $3 \sigma$ detection limits for both a $0.5^{\prime \prime}$ and $0.1^{\prime \prime}$ thin jet. These limits are derived from HST WFPC2 observations presented by Schwartz et al. (2000). In the second, most conservative case, the $\mathrm{BC}$ emission is still above the Spitzer sensitivity limit for the two shorter wavelength bands. However, the existing HST optical limits cannot be used to argue against a hadronic jet in this case. The angular length of the 'bridge' of PKS $0637-752$ is $\sim 8^{\prime \prime}$. This is easily resolved by HST. Most importantly, at $\lambda=3.6-8.0 \mu \mathrm{m}$, the most likely band for the BC scattered emission to appear, the Spitzer angular resolution $\left(\sim 1^{\prime \prime}-3^{\prime \prime}\right)$ is considerably smaller than the 'bridge' size, and we anticipate that Spitzer will resolve the $\mathrm{BC}$ emission along the 'bridge'.

\section{2. $3 C 273$}

The X-ray emission from knot A of $3 \mathrm{C} 273$ has been interpreted as both synchrotron (Marshall et al. 2001) and EC off the CMB (Sambruna et al. 2001). The discrepancy can be, at least partially, resolved by studying the spectrum of the near IR - optical - UV emission. HST and VLA observations by Jester et al. (2002) showed that the near IR - optical - UV spectrum of knot $A$ is flatter than the radio - near IR spectrum, indicating the presence of a high energy component, which, they suggest, can be interpreted either as EC off the $\mathrm{CMB}$ or as a second, synchrotron component from an independent high energy electron population. The constraints on the jet power and matter content depend on the interpretation we adopt.

EC off the CMB. We examine first the possibility that the $\mathrm{X}$-rays in knot $\mathrm{A}$ are due to $\mathrm{EC}$ off the CMB. We adopt again $\gamma_{\min }=20$, which, using Equation (15) and data from Marshall et al. (2001) listed in Appen$\operatorname{dix} \mathrm{A}$, corresponds to a minimum power Doppler factor $\delta_{\min }=16.6$ for a leptonic and $\delta_{\min }=26.5$ for a hadronic composition (these values of $\delta$ are significantly higher those inferred from observations of superluminal motions ( $\delta \sim 10$; Pearson et al. 1981) assuming $\delta=\Gamma$, a problem we discuss in $\S 6$ ). This corresponds to a jet minimum power $L_{\min }=3.3 \times 10^{45} \mathrm{erg} \mathrm{s}^{-1}$ for a leptonic jet and $L_{\min }=2.1 \times 10^{46} \mathrm{erg} \mathrm{s}^{-1}$ for a hadronic jet. The corresponding lepton power is $L_{\text {lept }}=1.6 \times 10^{45} \mathrm{erg}$ $\mathrm{s}^{-1}$ for a leptonic jet and $L_{\text {lept }}=2.9 \times 10^{44} \mathrm{erg} \mathrm{s}^{-1}$ for a hadronic jet. As in the case of PKS 0637-752, these numbers are only weakly affected by our choice of $\gamma_{\min }$, as long as $\gamma_{\min } \gtrsim 10$. The deprojected 'bridge' length, derived under the assumption $\theta=1 / \Gamma$ (at the redshift of the source, $z=0.158,1^{\prime \prime}$ corresponds to $2.7 \mathrm{Kpc}$ ) is $l \approx 580 \mathrm{Kpc}$ for a leptonic jet and $l \approx 930 \mathrm{Kpc}$ for $\mathrm{a}$ hadronic jet.

Using these values for the Doppler factor and 'bridge' length we calculate the $\mathrm{BC}$ flux for an $e-p$ and an $e^{ \pm}$ 
composition for both cases A and B for the cold lepton power. As can be seen in Figure 6, the situation is similar with that in PKS 0637-752. The BC emission of a leptonic jet peaks at mid IR energies, while that of a hadronic jet at near IR - optical energies. In case $A$, the anticipated mid IR flux is well above the Spitzer sensitivity limits for both compositions; the hadronic composition for case $\mathrm{A}$, however, violates the $H S T 3 \sigma$ detection limits for both a $0.5^{\prime \prime}$ and $0.1^{\prime \prime}$ thin jet (limits derived from 2001 July 17 th STIS observations at $7219 \boldsymbol{A}$, exposure tume $6073 \mathrm{~s}$, f $28 \times 50$ long pass filter, P.I. Meg Urry ). In the most conservative case B, the $\mathrm{BC}$ emission is still above the Spitzer sensitivity limit for the two shorter wavelength bands. The expected optical emission is lower than the HST $3 \sigma$ detection limits for both compositions, offering no additional constraints. As in PKS 0637-752, the large angular size of the 'bridge' $\left(\sim 13^{\prime \prime}\right)$ guarantees that the BC emission of the 'bridge' can be resolved by Spitzer.

Synchrotron. We turn now to the synchrotron interpretation. Using Equations (19, 20), we calculate the jet minimum power as a function of $\gamma_{\min }$. An upper limit on $\gamma_{\min }$ is set by the requirement that the lowest energy electrons are energetic enough to produce the lowest observed synchrotron emission at $\nu_{s, \mathrm{~min}}=408$ $\mathrm{MHz}$ (Foley \& Davis 1985) associated with knot A: $h \nu_{s, \min } / m_{e} c^{2}<y_{\min } \gamma_{\min }^{2}(1+z)$. As can be seen in Figure 7, in the case of a leptonic composition the minimum power required in the leptonic component (thin dashed line) drops as $\gamma_{\min }$ increases, down to $\approx 4 \times 10^{44}$ erg $s^{-1}$, a factor of $\approx 4$ below the minimum power in the leptonic component under the EC interpretation. In the case of a hadronic composition, the minimum power required in the leptonic component (thick dashed line) also decreases with $\gamma_{\min }$; even at its lowest value, however, it remains more powerful than the the minimum power in the leptonic component under the EC interpretation. Note that the difference in leptonic power between the leptonic and the hadronic compositions decreases with increasing $\gamma_{\min }$ as the energy per electron gradually becomes comparable to the proton rest mass energy.

Calculating the $\mathrm{BC}$ component in the synchrotron case requires a choice of $\delta$. Small values of $\delta$ will render the $\mathrm{BC}$ emission undetectable, since $L_{B C} \propto \delta^{3}$. Adopting the same values for $\delta$ as those derived through in the EC off the CMB case, results in detectable by Spitzer BC emission for both leptonic and hadronic jet compositions in case $A$. In case $B$, the $B C$ flux can drop below the Spitzer detectability limits, because the large permitted values of $\gamma_{\min }$ reduce the power of the cold lepton beam $\left(L_{e}=L_{\text {lept }}(p-2) /(p-1) \gamma_{\text {min }}\right)$.

\section{DISCUSSION AND CONCLUSIONS}

In the preceding sections we have formulated and examined the process of bulk Comptonization of the CMB photons by the 'cold' electrons of the relativistic flows of Chandra-detected extragalactic jets.

The physical process of $\mathrm{BC}$ scattering of the CMB is certainly taking place, as long as there are cold electrons propagating in a jet. One, however, has to focus on systems where the BC signature is expected to be (i) strong and (ii) minimally contaminated by other emissions. We argued that these conditions are favorably met in the Chandra - detected superluminal quasars
PKS 0637-752 and 3C 273, sources in which the jet radiates very weakly in radio, optical, and $\mathrm{X}$-ray energies for $\sim 10^{\prime \prime}$ between the core and the first knot. Based on the fact that the 'bridge' connecting the core to the first knot is only weakly radiating, we argued that the leptons in this radiatively inefficient section of the jet are transported practically cold. We then calculated the power and Doppler factor of the flow required in the first knot to produce the observed broadband spectrum under minimum energy conditions (DA04), adopting EC scattering of the CMB by relativistically moving plasma as the $\mathrm{X}$-ray emission mechanism. For $3 \mathrm{C} 273$ we also examined the possibility that the $X$-ray emission is due to synchrotron, a viable alternative for this source.

Using these power and Doppler factor estimates, we calculated the $\mathrm{BC}$ emission for an $e-p$ and $e^{ \pm}$jet composition, in each case considering two ways for energizing the electrons in the knot: in case $A$ we assumed that the lepton power needed in the knot is provided by the cold leptons in the beam alone, while in the most conservative case $B$ that the jet provides simply the number of leptons needed to produce the knot emission (while the required power is provided by another agent, i.e. protons, magnetic fields). The resulting $\mathrm{BC}$ mid IR emission is above the Spitzer detectability limits in both cases and for both compositions, and actual Spitzer measurements of the 'bridge' mid IR emission, together with optical - near IR observations, possibly including near IR polarimetry will measure or, at worst, substantially constrain the matter content of these jets. As we showed in $\$ 2$, a measurement of the polarization of the $\mathrm{BC}$ component, together with an estimate of its peak frequency, can break the degeneracy between the orientation of the jet and its bulk Lorentz factor.

Existing HST limits for both 3C 273 and PKS 0637-752 already disfavor case $\mathrm{A} e-p$ models, in agreement with similar conclusions from blazar studies Sikora \& Madejski (2000). Additional constraints for pure $e-p$ jets come from the large Lorentz factors required. Although values of $\delta_{\min } \sim 30$ are still compatible with the apparent superluminal motions observed in some blazars (e.g. Jorstad et al. 2002), the number of such highly relativistic sources should not overproduce the parent (misaligned) source population (e.g. Lister 2003). Additionally, as we mentioned in $\$ 5.1,5.2$, the large Doppler factors required for pure $e-p$ jets, suggest jet length over 1 Mpc long, a value barely compatible with the largest jets of known radio galaxies (e.g. Subrahmanyan, Saripalli, \& Hunstead 1996).

Measuring the $\mathrm{BC}$ emission of the 'bridge' can be used to measure what fraction of the cold electrons propagating in the 'bridge' are picked up by the particle acceleration mechanism in the knot and are accelerated to high energies. This "injection efficiency" of particle acceleration is, so far, a theoretically not well understood and observationally not strongly constrained quantity (e.g. Gallant 2002).

A failure to detect the $\mathrm{BC}$ emission has definite implications for the matter content of jets; however, given the limited sensitivity of Spitzer, such a nondetection will leave several possibilities open. For $3 \mathrm{C} 273$, where a synchrotron interpretation of the knot X-ray emission is a plausible alternative, a non-detection would still be com- 
patible with synchrotron X-ray emission in Case B. A possible source of the separate high energy electron population required could be the decay of a neutron beam (Dermer \& Atoyan 2004b). If the knot X-ray emission is $\mathrm{EC}$ in nature (this mechanism is plausible for $3 \mathrm{C} 273$ and favored for PKS 0637-752, as we discuss in §5), deviations from equipartition (e.g. Kataoka \& Stawarz 2004) and/or from the adopted orientation $\theta=1 / \Gamma$ can reduce the BC flux below the Spitzer detection limits in case B, in which case we will be able set an upper limit on the number of cold leptons in the jet as a function of $\delta$. We note here that the minimum power needed in the EC model is already $\gtrsim 10^{46} \mathrm{erg} \mathrm{s}^{-1}$ and one cannot deviate significantly from that without requiring jet powers greater than the Eddington luminosity of a $10^{9} M_{\odot}$ black hole. If significant deviations from equipartition are needed to explain future non-detection of the BC emission by Spitzer, they will impose severe constraints on the EC knot emission model, particularly in the case of the higher power hadronic jets. ***** Even if Spitzer does not detect the $\mathrm{BC}$ emission, our method can still be applied in the near future using the JWST; this instrument is expected to be $\sim 3$ orders of magnitude more sensitive than Spitzer and therefore probe much fainter levels of $\mathrm{BC}$ emission.

An assumption made in our calculations is that the Doppler factor of the jet flow in the 'bridge' between the core and the knot is the same as the Doppler factor of the knot. This can happen if the flow does not decelerate substantially at the knot, which can happen if the knot is the site of an oblique shock. Note, however, that if the knot is a separate entity propagating in the jet, as in one of the cases examined by Tavecchio et al. (2003) and Stawarz et al. (2004), the physical properties of the knot will be unrelated to those of the "bridge" and our method will not applicable for the determination of any of the jet parameters. In the case of PKS 0637-752, VLBI observations of superluminal velocities with $v_{a p p}=17.8 \pm 1 c$ in the core of the source (Lovell et al. 2000) set limits for $\Gamma>17.8, \theta<6^{\circ} .4$, in agreement with the Doppler factor $\delta=17.4$ derived from minimizing the jet power in an $e^{ \pm}$jet. Similar values for the Doppler factors in the core and the first knot were also derived by Tavecchio et al. (2004) using spectral modeling of both the core and the first knot for blazars PKS 1510-089 and 1641+399 and by Jorstad \& Marscher 2004 for the superluminal source
$0827+243$; these authors concluded that there is no bulk flow deceleration between the core and the first knot in these sources. The situation, however, seems different for 3C 373, where the superluminal velocities observed in the VLBI core suggest $\Gamma \sim 10$ (e.g. Pearson et al. 1981), significantly lower than the Doppler factor $\delta=16.6$ needed for an $e^{t}$ jet in minimum power. This leaves open the possibility for a synchrotron interpretation of the X-ray emission, which, as we argued in $\S 4.2,5.2$, does not allow for a unique determination of $\delta$ and, subsequently for a firm estimate of the $\mathrm{BC}$ emission.

We have also assumed in our calculations the leptons in the radiatively inefficient 'bridge' between the core and the first knot are cold $(\langle\gamma\rangle \approx 1)$. Considering that electrons in the large scale jet cannot cool down to $\gamma \sim 1$ in the flow frame once accelerated to relativistic energies, the cold electrons in the "bridge" region, in the situation we envisage, are cold not because they have been radiatively or adiabatically cooled down, but because they have never been accelerated. In fact, this is in agreement with our understanding of stochastic particle acceleration, according to which, generally, only a small fraction of the available particles is accelerated to high energies. Clearly there are non-thermal electrons, since the broadband emission in the 'bridge' of both PKS 0637-752 and 3C 273 is weak but not absent. However the majority of the electrons must be at Lorentz factors smaller than those required to obtain EC off the CMB at optical energies. For $\Gamma \approx 10$ this implies $\gamma \lesssim 4$. If the electrons are not really cold, but they have a distribution around some small $\gamma$, because the electron losses scale as $\gamma^{2}$, the power and the peak frequency of the bulk Compton emission would be higher by the same factor. Our assumption, therefore, represents a lower limit on the expected $\mathrm{BC}$ emission.

As was discussed by Schwartz (2002) the knot X-ray emission due to EC off the CMB will remain visible at the same flux level independent of redshift. This is also the case for the $\mathrm{BC}$ emission from cold leptons in relativistic jets. This suggests an exciting possibility for jets that have a very low radiative efficiency past the core (practically sources like PKS 0637-752, but without the acceleration events that produce the broadband non-thermal knot emission): their IR-optical BC emission will be detectable independent of redshift, and it will be the only observable signature of these otherwise invisible jets.

\section{APPENDIX}

\section{FUNCTIONS AND OBSERVABLES}

We reproduce here the functions $f_{1}, f_{2}, f_{3}, f_{4}$ that result from the formalism of DA04. The function $f_{1}$ appears in Equation (15) - eq. (12) of DA04 - that relates the minimum power Doppler factor $\delta_{\min }$ to the knot matter content as expressed through $k_{p e}$ and the minimum Lorentz factor $\gamma_{\min }$ of the electron distribution:

$$
\begin{gathered}
f_{1}=\left(\frac{9(1+p) d_{L}^{2} m_{e} c^{2}(1+z)^{(p-3) / 2}}{8(p-2) c \sigma_{\tau} r_{b}^{3}}\right)^{1 /(5+p)}\left[\frac{\left(f_{\epsilon_{E C}}^{E C}\right)^{5+p} u_{B_{c c}}^{3-p}}{\left(f_{\epsilon_{s}}^{s}\right)^{4} u_{\star}^{p+5}}\right]^{1 /(1+p)(5+p)} \times \\
\epsilon_{s}^{2(3-p) /(p+1)(p+5)} \times\left(\frac{2 \epsilon_{\star}}{\epsilon_{E C}}\right)^{(3-p) / 2 /(p+1)} .
\end{gathered}
$$


$f_{2}$ arises in the calculation of minimum jet power, by combining eq. (11) of DA04 for the particle energy content with the expression $L_{\text {part }}=\pi r_{b}^{2} \Gamma^{2} c W_{\text {part }}^{\prime}$ and setting $\Gamma=\delta=\delta_{\min }$ :

$$
f_{2}=\frac{9 \pi m_{e} c^{2} d_{L}^{2} f_{\epsilon_{E C}}^{E C}}{2 \sigma_{\tau} u_{\star} r_{b}(p-2)}\left(\frac{(1+z) \epsilon_{E C}}{2 \epsilon_{\star}}\right)^{(p-3) / 2}
$$

$f_{3}$ arises in the calculation of minimum jet power, by combining eq. (10) of DA04 for the magnetic field with the expression $\dot{L}_{B}=\pi r_{b}^{2} \Gamma^{2} c W_{B}^{\prime}$ and setting $\Gamma=\delta=\delta_{\text {min }}$ :

$$
f_{3}=\pi r_{b}^{2} c u_{B_{\mathrm{cr}}}\left(\frac{f_{\epsilon_{s}}^{s} u_{\star}}{f_{\epsilon_{E C}}^{E C} u_{B_{\mathrm{cr}}}}\right)^{4 /(p+1)}\left(\frac{\epsilon_{E C}}{2 \epsilon_{s} \epsilon_{\star}}\right)^{2(3-p) /(p+1)} .
$$

$f_{4}$ appears in Equation 18 (eq. (8) of DA04):

$$
f_{4}=\left[\frac{9 m_{e} c^{2} d_{L}^{2} f_{e s}^{s} \epsilon_{s}^{(p-3) / 2}}{2 c \sigma_{\tau} u_{B_{c r}}^{2}(p-2) r_{b}^{3}}\right]^{2 /(5+p)} .
$$

These functions depend on the following quantities:

$z$, the source redshift

$d_{L}$, the source luminosity distance

$p>2$, the electron index, related to the radio and X-ray spectral index $\alpha=(p-1) / 2$

$r_{b}$, the knot radius

$u_{B_{c r}}=B_{c r}^{2} / 8 \pi=7.7510^{25} \mathrm{erg} \mathrm{cm}^{-3}$, where $B_{c r}=m_{e}^{2} c^{3} / e \hbar$ is the critical magnetic field

$u_{\star}=410^{-13}(1+z)^{4}$ erg s$^{-1}$, the CMB photon energy density at $z$

$\epsilon_{\star}=2.70 k T_{C M B}(1+z) / m_{e} c^{2}=1.2410^{-9}(1+z)$, the dimensionless CMB photon energy at $z$

$\epsilon_{s}=h \nu_{s} / m_{e} c^{2}$, where $\nu_{s}$ is the radio (synchrotron) observation frequency

$\epsilon_{E C}=h \nu_{E C} / m_{e} c^{2}$, where $\nu_{E C}$ is the X-ray (EC) observation frequency

$f_{\epsilon_{s}}^{s}=\nu_{s} f_{\nu_{s}}$, where $f_{\nu_{s}}$ is the observed radio flux

$f_{\epsilon E C}^{E C}=\nu_{E C} f_{\nu_{E C}}$, where $f_{\nu_{E C}}$ is the observed X-ray flux.

In the case of PKS 0637-752 ( $z=0.651$ corresponding to $d_{L}=1.910^{28} \mathrm{~cm}$ or to $6.9 \mathrm{kpc}$ per arcsecond) the following values for the knot W7.8, taken by Schwartz et al. (2000) and by Chartas et al. (2000), were used in $\S 4$ (used also by DA04): $\nu_{s}=4.8 \mathrm{GHz}, f_{\nu_{s}}=54 m J y, \alpha_{r}=0.8, \nu_{E C}=3.810^{17} \mathrm{~Hz}, f_{\nu_{E C}}=6.610^{-9} \mathrm{Jy}$. The knot W7.8 is not resolved in the optical and it is assumed that $r_{b}=1 \mathrm{Kpc}$, corresponding to a knot diameter of $\sim 0.3^{\prime \prime}$.

For 3C $273,\left(z=0.158\right.$ corresponding to $d_{L}=2.310^{27} \mathrm{~cm}$ or to $2.7 \mathrm{kpc}$ per arcsecond $)$ the following values for knot A, taken by Marshall et al. (2000) were used in $\S 4: \nu_{s}=1.65 \mathrm{GHz}, f_{\nu_{o}}=0.42 J y, \alpha_{r}=0.76, \nu_{E C}=2.410^{17} \mathrm{~Hz}$, $f_{\nu_{E C}}=3.810^{-8} \mathrm{Jy}$. For knot A, $r_{b}=1 \mathrm{Kpc}$ is assumed, corresponding to a knot diameter of $\sim 0.75^{\text {“. }}$.

\section{REFERENCES}

Aharonian, F. A. 2002, MNRAS, 332, 215

Begelman, M. C. \& Sikora, M. 1987, ApJ, 322, 650

Blandford, R. D. \& Znajek, R. L. 1977, MNRAS, 179, 433

Blandford, R. D. \& Payne, D. G. MNRAS, 1982, 199, 883

Celotti, A. \& Fabian, A. C. 1993, MNRAS, 264, 228

Celotti, A., Ghisellini, G. \& Chiaberge M. 2001, MNRAS, 321, L1

Chartas, G. et al. 2001, ApJ, 542, 655

Cheung, C. C. 2004 , ApJ, 600 , L23

Dermer, C. D. \& Atoyan, A. M. 2002, ApJ, 568, L81

Dermer, C D. \& Atoyan, A. 2004a, ApJ, 611, L9 (DA04)

Dermer, C D. \& Atoyan, A. 2004b, ApJ, 613, 151

Foley, A. R. \& Davis, R. J. 1985, MNRAS, 216, 679

Gallant, Y. A. 2002, in Relativistic Flows in Astrophysics, eds.

A.W. Guthmann, M. Georganopoulos, A. Marcowith, \& K.

Manolakou, Lecture Notes in Physics, vol. 589.

Georganopoulos, M. \& Kazanas, D. 2003, ApJ, 589, L5

Georganopoulos, M. \& Kazanas, D. 2004, ApJ, 604, L81

Ghisellini, G., \& Celotti, A. 2001, MNRAS, 327, 739

Gizani, A. B. N. \& Leahy, J. P. 2004, MNRAS, 350, 865

Hirotani, K. 2004, ApJ, in press, also in astro-ph/411087

Jester, S., Röser, H.-J., Meisenheimer, K., \& Perley, R. 2002, A\&A, $385, \mathrm{~L} 27$

Jorstad, S. G., Marscher, A. P., Mattox, J. R., Aller, M. F., Aller,

H. D., Wehrle, A. E., \& Bloom, S. D. 2002, ApJ, 556, 738

Jorstad, S. G. \& Marscher, A. P. 2004, ApJ, 614, 615

Kataoka, J, \& Stawarz, L. 2004, ApJ, in press, also in astro$\mathrm{ph} / 0411042$

Kirk, J. G., Guthmann, A. W., Gallant, Y. A., \& Achterberg, A. ApJ, 542, 235

Königl A. 1989, ApJ, 342, 208
Lister, M. L. 2003, ApJ, 599, L105

Lovell, J. E. J. et al. 2000, in Astrophysical Phenomena Revealed by Space VLBI, ed. H. Hirabayashi, P. G. Edwards, \& D. W. Murphy (Sagamihara: ISAS), 215

Marshall, H. L. et al. 2001, ApJ, 549, L167

Marshall, H. L. et al. 2004, ApJ, in press

Martell, A. R. et al. 2003, AJ, 125, 2964

Moderski, R., Sikora, M., Madejski, G. M., \& Kamae, T. 2004, ApJ, 611, 770

Omma, H. \& Binney, J. 2004, MNRAS, 350, L13

Pearson, T. J. et al. 1981, Nature, 290, 365

Reynolds, C. S., Fabian, A. C., Celotti, A. \& Rees, M. J. 1996, MNRAS, 283, 873

Rybicki, G. R. \& Lightman, A. P. 1979, Radiative Processes in Astrophysics (Willey: New York)

Sambruna, R. M., Urry, M. C., Tavecchio, F., Maraschi, L., Scarpa, R., Chartas, G., \& Muxlow, T. 2001, ApJ, 549, L161

Sambruna, R. M., Maraschi, L., Tavecchio, F., Urry, M. C., Cheung, C. C., Chartas, G., Scarpa, R., \& Gambill, J. K. 2002, ApJ, 571, 206

Sambruna, R. M., Gambill, J. K., Maraschi, L., Tavecchio, F., Cerutti, R., Cheung, C. C., Urry, M. C., \& Chartas, G. 2004, ApJ, 608, 698

Schwartz, D. E. et al. 2000, ApJ, 540, L69

Schwartz, D. E. 2002, ApJ, 569, L23

Siemiginowska, A., Bechtold, J., Aldcroft, T. L., Elvis, M., Harris, D. E., \& Dobrzycki, A. 2002, ApJ, 570,543

Siemiginowska, A., Smith R. K., Aldcroft T. L., Schwartz, D. A., Paerels, F., \& Petric, A. O. 2003, ApJ, 598, L15 
Sikora, M., Madejski, G., Moderski, R. \& Poutanen, J. 1997, ApJ, 484,108

Sikora, M. \& Madejski, G. 2000, ApJ, 534, 109

Spergel, D. N. et al. 2003, ApJS, 148, 175

Stawarz, L., Sikora, M., Ostrowski, M., \& Begelman, M. C. 2004, ApJ, 608, 95

Subrahmanyan, R., Saripalli, L., \& Hunstead, R. W. 1996, MNRAS, 279, 257

Tavecchio, F., Maraschi, L., Sambruna, R. \& Urry, C. M., 2000, ApJ, 544, L23

Tavecchio, F., Ghisellini, G., \& Celotti, A. 2003, A\&A, 403, 83
Tavecchio, F., Maraschi, L., Sambruna, R. M., Urry, C. M., Cheung, C. C., Gambill, J. K., \& Scarpa, R. 2004, ApJ, 614, 64

Wardle, J. F. C., Homan, D. C., Ojha, R., \& Roberts, D. H. 1998, Nature, 395, 457

Urry, C. M. \& Padovani, P. 1995, PASP, 107, 803

Yuan, W., Fabian, A. C., Celotti, A., \& Jonker, P. G. 2003, MNRAS, 346, L7 